\title{
Properties of LTCC Dielectric Tape in High Temperature and Water Environment
}

\author{
S. Toskov, A. Maric, N. Blaz, G. Miskovic, and G. Radosavljevic
}

\begin{abstract}
This paper focuses on development of sensors, fabricated in Low Temperature Co-fired Ceramics (LTCC) technology. First sensor is developed for extremely high temperature characterization of LTCC material. Additionally, the influence of used numbers of substrate layers on overall capacitance of IDC has been investigated. Second sensor is developed for detection of liquid presence and quantity. Third sensor is developed for reliability monitoring of LTCC material in water environment. Sensors are planar, interdigital capacitors (IDC), designed and fabricated using CeramTec GC LTCC ceramic tapes as a carrying substrate. First investigation was directed towards temperature dependence of the permittivity and temperature dependence of the IDC capacitance up to $880^{\circ} \mathrm{C}$. The second one, shows effect of water penetration through LTCC ceramic material. The third investigation is conducted in order to demonstrate how long LTCC ceramic material keeps its properties while staying in water. Experimental results have proven reliability and good stability and sensitivity of analyzed LTCC ceramic material in high temperature and water environment.
\end{abstract}

Index Terms-Ceramic materials, IDC, LTCC, material properties.

\section{INTRODUCTION}

Low Temperature Co-fired Ceramic (LTCC) technology offers the possibility for multilayer circuit integration using single tape stacking process with printed conductive, dielectric and/or resistive pastes on it. These printed passive components on LTCC substrate offer 3D minimization of a whole structure. This technology can be used in different application areas such as space technology, radio technology, medicine, harsh operation environments, various industrial processes, construction industry etc. Knowledge on LTCC material properties is mandatory for proper design establishment in each specific area. Now days, one of the important application areas belong to environmental safety, where monitoring of different kinds and levels of pollutants is necessary. Especially in liquid environments, knowledge on sensor material properties is obligatory for achievement of reliability and stability of sensing systems. On the other hand, some future application areas require precise, high temperature characterization of LTCC material.

Therefore, high temperature characterization of CeramTec

Manuscript received November 24, 2012; revised February 28, 2013

S. Toskov, G. Miskovic, and G. Radosavljevic are with Institute of Sensor and Actuator Systems, Vienna University of Technology, Gusshausstrasse 27 - 29, Vienna, Austria (e-mail: sasa.toskov@tuwien.ac.at, goran.miskovic@tuwien.ac.at, goran.radosavljevic@tuwien.ac.at).

A. Maric and N. Blaz are with Faculty of Technical Sciences, University of Novi Sad, Trg Dositeja Obradovica 6, Novi Sad, Serbia (e-mail: amaric@uns.ac.rs,nelu@uns.ac.rs).
GC ceramic material has been realized in first research Temperature dependence of the permittivity and temperature dependence of the capacitance are determined. In order to show what happens if water penetration through LTCC ceramic material occurs, the second research is related to development of sensor for detection of water presence and quantity. The third research, related to the impact of water presence on properties of CeramTec GC LTCC ceramic tapes is presented. The aim was to examine durability of material properties preservation in water environment by daily monitoring of water penetration through the LTCC substrate.

Interdigital electrodes are one of the most commonly exploited periodic electrode structures that can be found in various electronic systems. IDC is used in many different sensor applications for detection of moisture, chemical compounds, gasses, pollution, pressure, motion, etc. [1]-[4]. Additionally, their simple design is suitable for fabrication in any presently available technology. Abovementioned facts set the IDC as a core of sensing structures in this research.

Following the Introduction, sensor design is presented in the Section II. Sensor fabrication and experimental results are presented in Section III. A short summary and drown conclusions are presented in Section IV.

\section{SENSOR DESIGN}

\section{A. Design of IDC}

IDC is a periodic electrode structure, of totally $N$ fingers, where each finger has width $w$, finger length $l$, adjacent finger spacing $s$, finger thickness $t$ with total area of $\left(d_{x} x d_{y}\right)$ $\mathrm{mm}^{2}$, and LTCC substrate of $h$ thickness. Layout of the designed IDC electrode and 3D models of sensors are presented in Fig. 1. Geometrical parameters of IDC are given in Table I. Sensor type-1 consist of a planar IDC electrode, screen printed at the half of the total height of the ceramic stack. Sensor type-2 has similar design as sensor type-1 where special interconnection technique was developed in order to expose sensor to extremely high temperature without losing connection with the measurement instrument. For sensor type-3 electrode is screen printed on the top of the four layer stack.

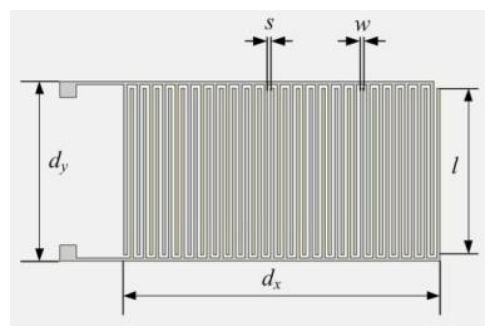

(a) Layout of IDC electrode 


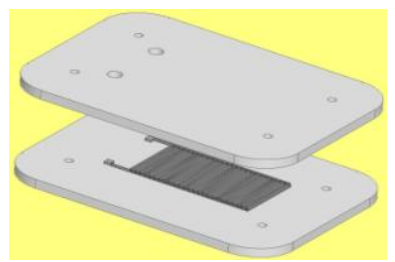

(b) 3D model of type-1 and type-2 IDC sensors

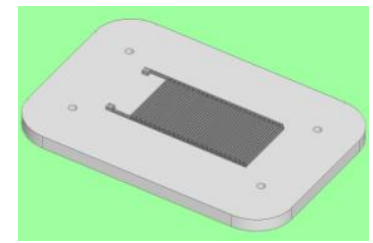

(c) 3D model of type-3 IDC sensor

Fig. 1. Sensor design

TABLE I: GEOMETRICAL PARAMETERS OF IDC ELECTRODE

\begin{tabular}{lc}
\hline \multicolumn{1}{c}{ Parameter } & Dimension \\
\hline Finger width $w[\mu \mathrm{m}]$ & 250 \\
\hline Finger spacing $s[\mu \mathrm{m}]$ & 250 \\
\hline Length of fingers $l[\mathrm{~mm}]$ & 13 \\
\hline finger thickness $t[\mu \mathrm{m}]$ & 12 \\
\hline Substrate height $h[\mu \mathrm{m}]$ & $480,900,1380,1780$ \\
\hline Number of fingers $N$ & 50 \\
\hline Total length $d_{x}[\mathrm{~mm}]$ & 24.75 \\
\hline Total width $d_{y}[\mathrm{~mm}]$ & 14 \\
\hline
\end{tabular}

\section{FABRICATION AND EXPERIMENTAL RESULTS}

\section{A. Fabrication of Sensors}

Sensors were fabricated using the standard LTCC technology. LTCC tapes in green state were formed into the final shape using laser micromachining, conductive IDC electrode was screen-printed using Heraeus TC7306A silver paste and then dried for 10 minutes at $80{ }^{\circ} \mathrm{C}$. Afterwards, LTCC tapes were stacked together and preheated at $80{ }^{\circ} \mathrm{C}$ for 30 minutes. Uniaxial lamination of preheated stack was performed with the load of $2200 \mathrm{~kg}$ at $75{ }^{\circ} \mathrm{C}$ temperature, in duration of $3 \mathrm{~min}$. Formed stack was sintered in the six-zone belt furnace, at peak temperature of $900{ }^{\circ} \mathrm{C}$ and $75 \mathrm{~min}$ total sintering cycle. Fabricated sensors are presented in Fig.2. For the sensor type- 1 wire connections have been soldered to the contact pads. For sensor type-2, wire connections could not be soldered to the IDC pads. Different joint technique had to be developed in order to expose sensor to extremely high temperature without losing connection with the measurement instrument. For that purpose, silver wires were used and fed through channels to reach the IDC pads. Afterwards, Heraeus TC7306A silver paste was applied on both, pads and silver wires. Interconnection bonding technique was executed at peak temperature of $700{ }^{\circ} \mathrm{C}$ over $30 \mathrm{~min}$. Sensor type-3 uses the same standard soldering process for connecting the wires to pads as for sensor type- 1 .

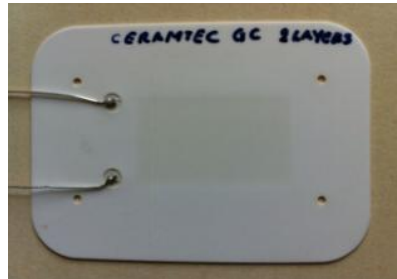

(c) type-1

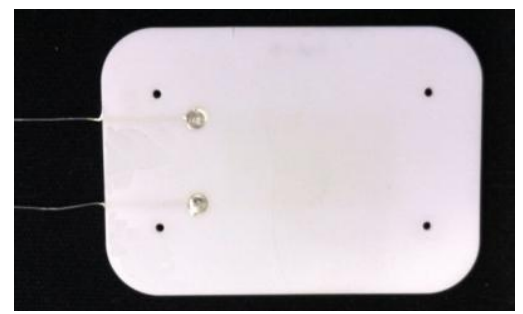

(a) type-2

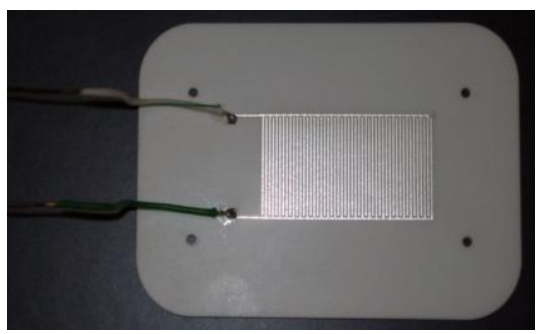

(b) type-3

Fig. 2. Fabricated sensors

\section{B. IDC Capacitance Dependence on Substrate Thickness}

Capacitance dependence of IDC on number of used ceramic layers has been investigated. Four sensors type-1 were fabricated using CeramTec GC ceramic tapes with 2, 4, 6 and 8 layers with total sintered thickness of $480 \mu \mathrm{m}, 900$ $\mu \mathrm{m}, 1380 \mu \mathrm{m}$ and $1780 \mu \mathrm{m}$, respectively. Measured results have shown that for higher numbers of used layers higher capacitance is achieved in air environment, Fig.3. However, for structures with 4 layers, capacitance reaches its saturated value, and starts to be practically independent on additional number of layers used. Results can be explained with the fact that with increased number of ceramic layers more lines of the capacitor electric field close through the medium with higher permittivity than air, where $\varepsilon_{r}=7.9+/-0.6$ [5] and $\varepsilon_{r}=1$, for CeramTec GC and air, respectively. According to Igreja [6] this occurs when $h=\lambda / 2$, where $h$ is the height of the substrate from the electrode plane and $\lambda=2(w+s)$ is the sensor wavelength, where $w$ is finger width and $s$ is adjacent finger spacing. For this IDC, $\lambda=1000 \mu \mathrm{m}$. That means that sensor is not sensitive to distance from the electrode plane greater than about half the sensor wavelength, which is in this case $500 \mu \mathrm{m}$. Saturation dependence on number of used layers for designed IDC is presented in Table II.

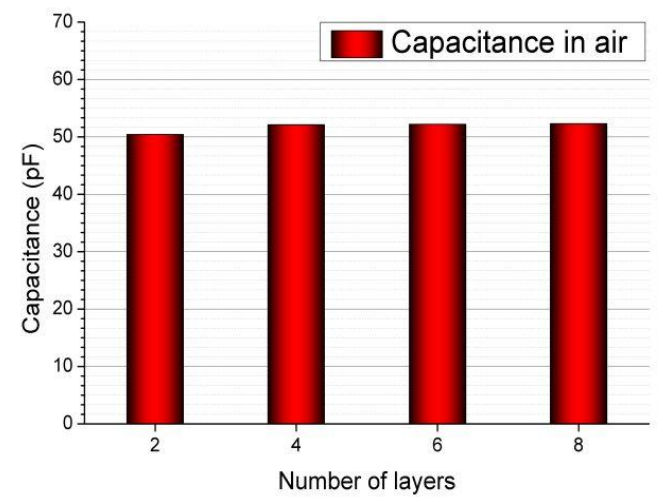

Fig. 3. IDC capacitance dependence on substrate thickness in air for CeramTec GC. 
TABLE II: SATURATION DEPENDENCE ON SUBSTRATE HEIGHT IN AIR

\begin{tabular}{|c|c|c|c|c|c|c|}
\hline \multirow{2}{*}{} & $\begin{array}{c}\text { No. of } \\
\text { layers }\end{array}$ & $\begin{array}{c}\text { Total } \\
\text { height } \\
{[\mu \mathrm{m}]}\end{array}$ & $\begin{array}{c}\mathrm{h} \\
{[\mu \mathrm{m}]}\end{array}$ & $\begin{array}{c}\lambda / 2 \\
{[\mu \mathrm{m}]}\end{array}$ & $\mathrm{C}[\mathrm{pF}]$ & $\begin{array}{c}\text { Satu } \\
\text { rati } \\
\text { on }\end{array}$ \\
\hline \multirow{4}{*}{$\begin{array}{c}\text { CeramTec } \\
\text { GC }\end{array}$} & 2 & 480 & 240 & 500 & 50.42 & \\
\cline { 2 - 8 } & 4 & 900 & 450 & 500 & 52.11 & $*$ \\
\cline { 2 - 8 } & 8 & 1380 & 690 & 500 & 52.21 & \\
\cline { 2 - 8 } & 8 & 1780 & 890 & 500 & 52.29 & \\
\hline
\end{tabular}

\section{High Temperature Characterization of CeramTec GC}

High temperature characterization of CeramTec GC ceramic material was directed towards temperature dependence of the permittivity and temperature dependence of the capacity.

For temperature dependence of the permittivity, sample discs with $10 \mathrm{~mm}$ diameter with double sided metallization were prepared. The capacitance was measured at temperatures up to $850{ }^{\circ} \mathrm{C}$. Permittivity was calculated from the measured capacitance values of the samples and their geometry. Assumption in calculation was that capacitor is circular disc of ceramic material without thermal expansion interference from the applied electrodes due to their extremely low thickness. Effects of stray fields at the border zones were neglected. Measurement set-up consists of a box furnace and LCR131 meter. Thermocouple probes were used for temperature measurement near the sample. Initial permittivity at room temperature is $\varepsilon_{r}=7,8$. Temperature dependence of the relative permittivity is presented in Fig. 4.

For temperature dependence of the capacity, type- 2 sensor with 8 layers was used. Reason why structure with 8 layers was chosen lays in fact that capacitance depends on geometrical parameters of IDC electrode and total substrate height. In case of CeramTec GC ceramic tapes, sensor reaches its saturated value first time for the structure with totally 4 layers and starts to be independent on permittivity of surrounding air. Thus, sensor with 8 layers was chosen in order to avoid any possible influence of permittivity changes over the temperature of surrounding air. The capacitance was measured for the temperatures up to $880{ }^{\circ} \mathrm{C}$. Measurement set-up, Fig. 5, consists of a box furnace, high frequency LCR meter Wayne Kerr 6500P, thermocouple probes near the sample and lap connected to thermometer for real time monitoring of temperature changes.

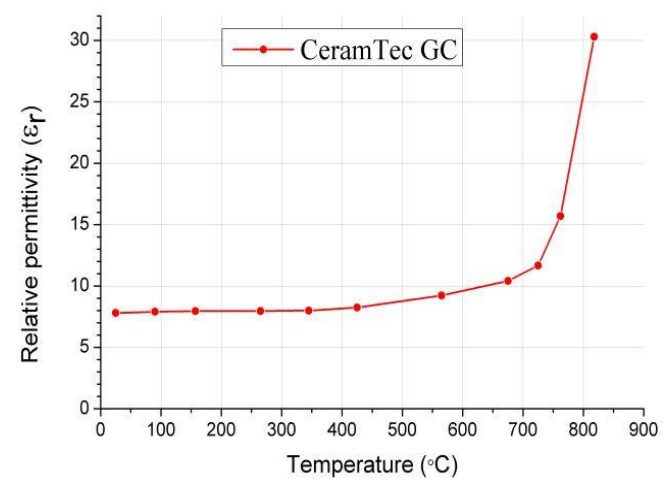

Fig. 4. Relative permittivity vs. temperature characteristics

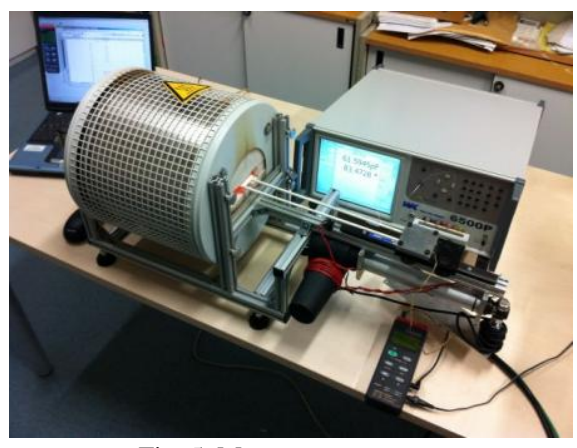

Fig. 5. Measurement set-up

Temperature dependence of the capacitance is shown in graph of Fig. 6. Dielectric properties are stable at lower temperatures up to $550{ }^{\circ} \mathrm{C}$, where relative difference between initial value at room temperature and measured values at elevated temperatures is below $10 \%$. Above indicated temperature, significant increase of CeramTec GC dielectric permittivity can be observed. For example, at $650{ }^{\circ} \mathrm{C}$ difference is $20 \%$ and for $750{ }^{\circ} \mathrm{C}$ capacitance is doubled.

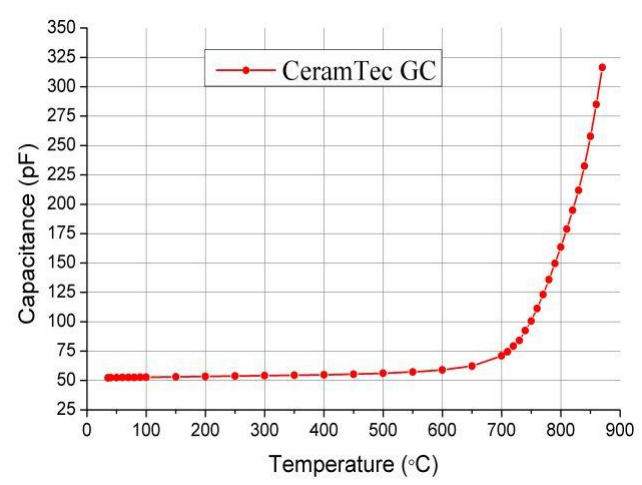

Fig. 6. Capacitance vs. temperature characteristics

\section{Liquid Presence and Quantity Test}

This experiment aims to examine what capacitance values of IDC can be expected if water penetration through the LTCC substrate occurs. For that purpose type-3 sensor was developed. Different amounts of water were placed on the sensor surface and capacitance frequency dependence was measured. Water volume varied in the range of $5 \mu \mathrm{l}$ to $30 \mu \mathrm{l}$, with increment of $5 \mu \mathrm{l}$. Water drops were distributed on the surface of IDC electrode and capacitance were measured. Obtained measures results are presented in Fig. 7. Repeated measurements with different distribution of single water drops proved that position of drops does not influence the capacitance values.

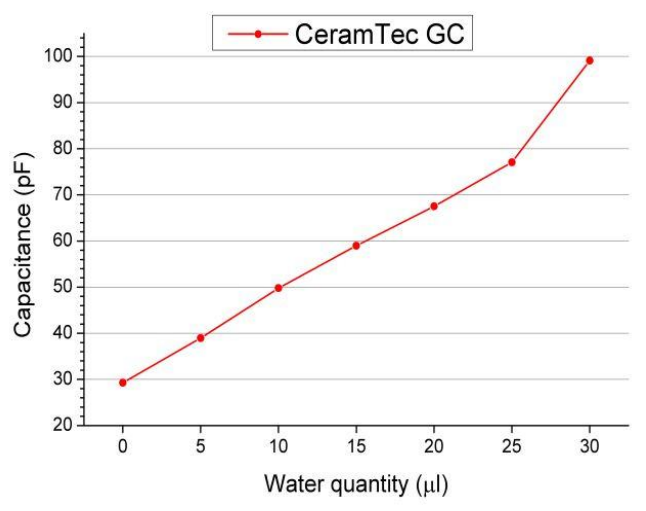

Fig. 7. Capacitance vs. water quantity 
Initial capacitance value in case when water is not applied is $29.26 \mathrm{pF}$. It can be observed from obtained results that small increment of water quantity causes capacitance value increment up to several times compared to its initial value. The same behavior can be expected if water penetration through LTCC substrate occurs.

\section{E. Long Term Reliability of CeramTec GC in Water Presence}

This experiment aims to examine reliability of CeramTec GC ceramic material in water environment by daily monitoring of water penetration through the LTCC substrate. In addition, if the water penetration occurs, after what time period would that happen?

Totally four sensors type- 1 with, 2, 4, 6 and 8 layers were tested. Sensors were set into $50 \mathrm{ml}$ of water and capacitances were recorded daily over 185 days, at room temperature. When sensor is placed in the water overall capacitance increases, due to higher permittivity of water comparing to air, $\varepsilon_{r}=80.1, \varepsilon_{r}=1$, respectively, Fig.8. As the number of LTCC layers increases, most of the electrical field penetrates through the LTCC substrate which has lower dielectric permittivity comparing to the water, decreasing overall capacitance. Measurement set up for daily capacitance monitoring is shown in Fig. 9. It consists of high frequency LCR meter Wayne Kerr 6500P and tested sensors type-1 inside the plastic glass. Capacitance of IDC will change if there is a change in the capacitive properties of the LTCC substrate or in its thickness. If CeramTec GC tapes have high porosity, water would penetrate through the substrate causing significant increment of overall capacitance as it was shown in Section D. During the first 65 days capacitances were measured daily. Afterwards capacitances were measured in a sequence of 10 days. The time dependence of capacitance changes, for IDC sensor with 2, 4, 6 and 8 layers is shown in Fig. 10. It was shown that capacitances remained unchanged in investigated time period. There was not significant increase of capacitance registered yet, indicating that water penetration through any of four tested structures didn't occur. Preliminary results during 185 days have proven reliability and good stability of CeramTec GC tape. It can be confirmed that this material is suitable for long term exploitation in water environment.

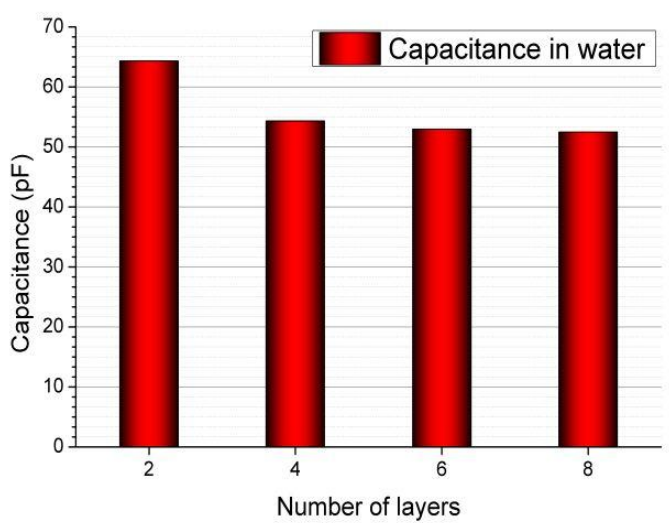

Fig. 8. IDC capacitance dependence on substrate thickness in water for CeramTec GC

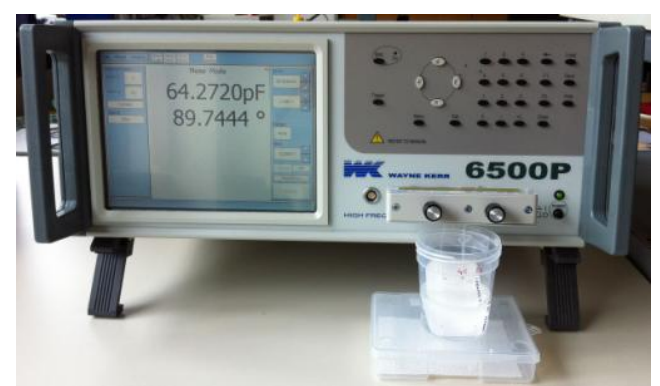

Fig. 9. Measurement set-up

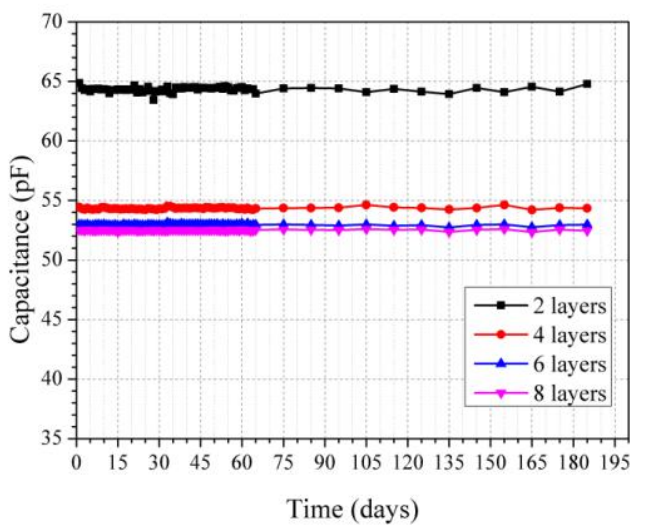

Fig. 10. Long term reliability of CeramTec GC tape in water environment

\section{CONCLUSIONS}

Three types of sensors were presented in this paper. All sensors consist of simple, planar interdigital capacitor, fabricated in the standard LTCC technology. Type-1 sensor was developed for capacitance dependence on the substrate thickness monitoring and the long term reliability monitoring of CeramTec GC ceramic material in water environment. Development of type-2 sensor was directed towards high temperature characterization of LTCC ceramic material.Type-3 sensor is developed for detection of water presence and quantity. . First research focuses on capacitance dependence on the substrate thickness and material properties as well as their change over temperature i.e. temperature dependence of the permittivity and temperature dependence of the capacitance. It was shown that for higher numbers of used layers higher capacitance is achieved in air environment. However, for structures with 4 layers, capacitance reaches its saturated value, and starts to be practically independent on additional number of layers used. In temperature dependence of the permittivity and temperature dependence of the capacity experiment was shown that dielectric properties are stable at lower temperatures up to $550{ }^{\circ} \mathrm{C}$. Up to this temperature, relative difference between initial values and measured values was below $10 \%$. Above this temperature, significant increase of CermTec GC ceramic material permittivity is observed. The second experiment showed what capacitance values can be expected if water penetration through the LTCC substrate occurs. It was shown that small increment of water quantity causes large increment of capacitance, indicating high sensitivity of the sensor. Repeated measurements with different distribution of single water drops proved that position of drops does not influence the capacitance values. 
The third experiment showed that water penetration through ceramic material didn't occur i.e. capacitances remained unchanged in investigated time period. Preliminary results during 185 days have proven reliability and good stability of CeramTec GC tape thus can be confirmed that this ceramic material is suitable for long term exploitation in water environment. Future work will focus on characterization of other commercially available LTCC dielectric tapes in high temperature and water environment.

\section{REFERENCES}

[1] P. A. Hochstein, U.S. Patent 6,094,981, 2000

[2] A. Quershi, Y. Gurbuz, W. P. Kang, and J. L. Davidson. A Novel Interdigitated Capacitor Based Biosensor for Detection of Cardiovascular Risk Marker. [Online]. Available: http://research.sabanciuniv.edu/13632/1/A_novel_interdigitated_capa citor_basedbiosensor_for_detection_of_cardiovascular_risk_marker.p df.

[3] A. R. M. Syaifudin, S. C. Mukhopadhyay, and P. L. Yu, "Modelling and Fabrication of Optimum Structure of Novel Interdigital Sensors for Food Inspection," Int. J. Numerical Modelling: El. Networks, Devices and Fields, Wiley Online Lib., vol. 25, pp. 64-81, 2012.

[4] A. Qureshi, J. H. Niazi, S. Kallempudi, and Y. Gurbuz, "Label-Free Capacitive Biosensor for Sensitive Detection of Multiple Biomarkers Using Gold Interdigitated Capacitor Arrays," Biosens. and Bioelectr., vol. 25, pp. 2318-2323, 2010

[5] LTCC Ceramtape-for Microhybrids and Bluetooth. [Online]. Available: http://www.ceramtec.de/files/el_ltcc_ceramtape.pdf

[6] R. Igreja and C. J. Dias, "Analytical Evaluation of the Interdigital Electrodes Capacitance for a Multi-Layered Structure," Sensors and Actuators A: Physical, vol. 112, no. 2-3, pp. 291-301, May 2004

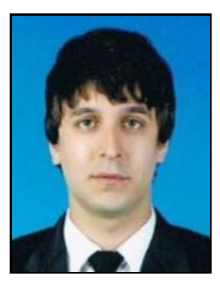

Sasa D. Toskov was born in Novi Sad, Republic of Serbia, in 1983. He received the M.Sc. degree in the field of electrical engineering from the Faculty of Technical Sciences, University of Novi Sad, Republic of Serbia in 2010. He joined the Institute of Sensor and Actuator Systems at Vienna University of Technology, Vienna, Austria in 2011 as a Project Assistant and now is working as an assistant and researcher. His main research interests are in the field of microelectronics and microelectronic technologies (LTCC), RF passive components (inductors, transformers), high frequency LTCC material characterization, design, modeling, simulation and fabrication of micro-heaters and micro-sensors.

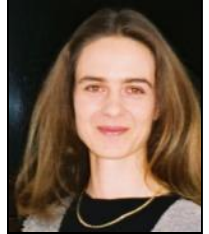

Andrea M. Marić (M’07) was born in Novi Sad, Serbia, in 1978. She received the B.S. and M.S. degree in the field of electrical engineering from the Faculty of Technical Sciences, University of Novi Sad, Serbia in 2005 and 2008 respectively. She joined the Faculty of Technical Sciences, Novi Sad in 2006 as a teaching assistant and now is working as an assistant and a researcher.

Her main research interests are in the field of microelectronics and microelectronic technologies (LTCC, PCB, flexible electronics), RF passive components (inductors, transformers), micro-sensors and high frequency material characterization. She has authored and coauthored more than 60 papers in scientific journals and conferences.

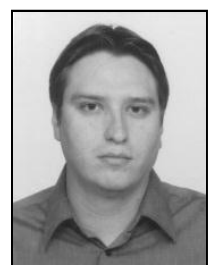

Nelu V. Blaž received the master's degree in 2005 , from the University of Novi Sad, Faculty of Technical Sciences, Novi Sad, Serbia. He is currently working on the $\mathrm{Ph} . \mathrm{D}$. thesis in electrical engineering specializing in microelectronics. Since 2007, he has worked in the Faculty of Technical Sciences, Department of Power, Electronics and Communication, Novi Sad as expert associate. His research interests include material characterization, RF measurements and micro-sensors.

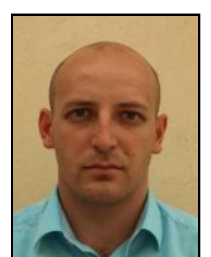

Goran Miskovic was born in 1988 in Novi Sad, Serbia. He received the B.Sc. degree in 2011 and M.Sc. degree in 2012 from Faculty of Technical Sciences, University of Novi Sad, Novi Sad, Serbia in the field of microelectronics. He is currently working towards the $\mathrm{Ph}$.D. degree in field of gas sensors in LTCC technology at the Vienna University of Technology, Vienna, Austria. His current research interests focus on chemical sensor and nanotechnology combined with LTCC technology.

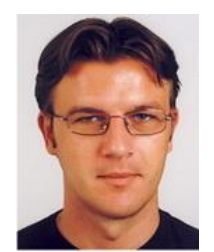

Technology.

His main research interests are in the field of microelectronics with the accent on design, modeling, simulation and fabrication of inductors, transformers and sensors in the LTCC and PCB technology. He has authored and coauthored more than 90 papers in scientific journals and conferences. 